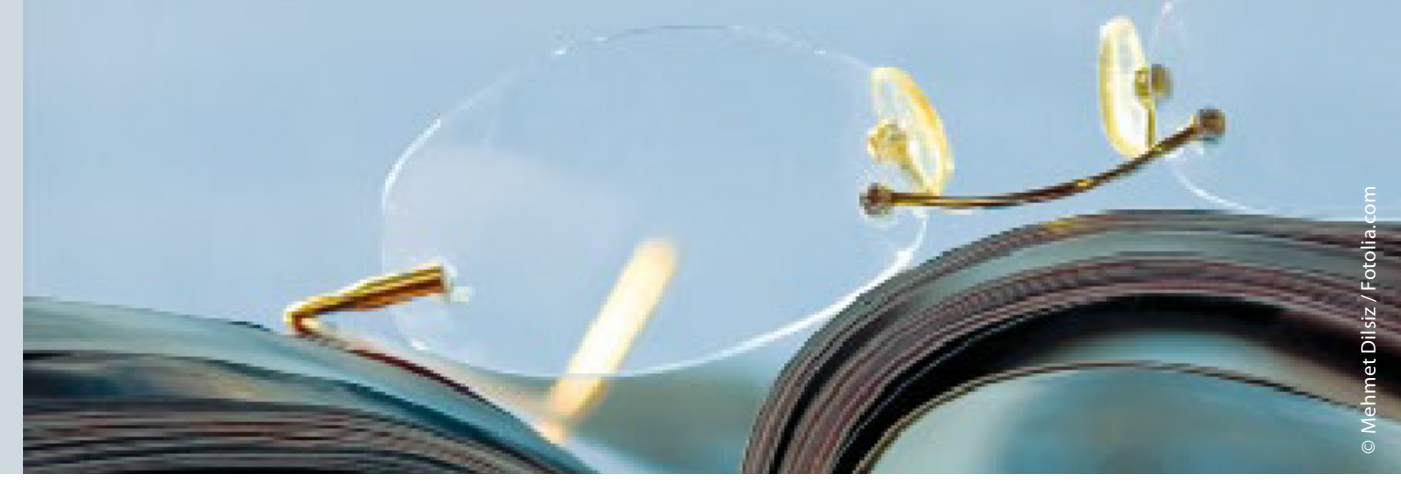

wachsenen. Patienten mit manischen Episoden hatten in nahezu allen erfassten Parametern des klinischen Schweregrades eine stärkere Beeinträchtigung als Patienten, die „nur“ an depressiven Episoden litten. Ob die Manie von Depressionen begleitet wurde oder nicht machte in dieser Hinsicht allerdings keinen Unterschied.

Kommentar: Mit dieser aufwändigen und groß angelegten epidemiologischen Studie können die Autoren die Hypothese, dass bipolare Störungen in der Jugend beginnen, erhärten. Die Autoren ziehen selbst die Schlussfolgerung, dass Früherkennungs- und Frühinterventionsprogramme für diese Population dringend notwendig sind. Ebenso unterstreicht diese Studie die Dringlichkeit einer besseren Zusammenarbeit von Erwachsenen- und Kinder-und-Jugendpsychiatrie, damit Jugendliche mit bipolaren Störungen nicht mit dem 18. Lebensjahr "verloren" gehen. Das an einigen Orten bereits begonnene Konzept der Adoleszenz-Zentren könnte hier modellhaft werden. Ein weiterer interessanter Aspekt der Studie ist die relative
Häufigkeit unipolarer Manien, der sicherlich weiterer Aufmerksamkeit bedarf. Schwierig hingegen erscheint, dass die Autoren gemischte Episoden nicht wirklich erfassen konnten - hier könnte noch ein gewisser verborgener Teil des Eisberges „Bipolare Störungen" treiben.

Prof. Dr. Helge Frieling

Merikangas KR et al. Mania with and without depression in a community sample of US adolescents. Arch Gen Psychiatry 2012; 69: 943-51

\title{
Sind Kinder von bipolaren Eltern häufiger psychisch krank?
}

Bipolare Störungen haben ein sehr hohes genetisches Risiko, daher erscheint es wahrscheinlich, dass Kinder von Elternteilen mit bipolarer Störung ebenfalls häufig daran erkranken. Es bleibt aber nicht bei bipolaren Störungen allein, wie eine aktuelle Arbeit belegt.

$\mathrm{D}$ ie Dutch Bipolar Offspring Study beobachtet seit 1997 eine Kohorte von Kindern mit mindestens einem bipolar erkrankten Elternteil. Die zu Studienbeginn zwischen 12 und 21 Jahre alten Probanden wurden nach einem, nach fünf und aktuell nach zwölf Jahren untersucht. Von der ursprünglichen Kohorte konnten in der aktuellen Untersuchung 108 Teilnehmer (77\%) erneut eingeschlossen werden. Drei von vier Kindern (72\%) erlitten im Beobachtungszeitraum eine manifeste psychische Störung (DSM IV Achse 1), davon 54\% eine affektive Störung. Störungen aus dem bipolaren Spektrum traten bei $13 \%$ der Studienteilnehmer auf, während nur $3 \%$ eine manifeste Bipolar-I-Störung erlitten. Bei dem größten Teil der Probanden mit Störungen aus dem bipolaren Spektrum begann die Erkrankung mit einer depressiven Episode. Etwa ein Viertel der Studienteilnehmer mit initial depressiven Episoden entwickelte eine Störung aus dem bipolaren Spektrum. Viele Probanden litten unter rezidivierenden Störungen (33\%) oder wiesen eine Komorbidität auf (67\%). Bei fast allen Probanden begann die Erkrankung vor dem 25. Lebensjahr. Über den Beobachtungszeitraum nahm der Anteil der Probanden, die völlig frei von psychischen Störungen war, dementsprechend kontinuierlich ab.
Kommentar: Die Stärke der vorliegenden Studie liegt in dem langen Beobachtungszeitraum und der dafür sehr niedrigen Ausfallrate. Dadurch können valide Aussagen nicht nur zum Untersuchungszeitpunkt, sondern auch im Langzeitverlauf getroffen werden. Für den postulierten hohen genetischen Background der bipolaren Störungen ist die Rate an Kindern mit bipolarer Störung erstaunlich niedrig. Allerdings passen diese Befunde auch gut zu der aktuellen Entwicklung in den großen genetischen Konsortien - hier wurden die interessantesten Befunde in der letzten Zeit bei der gemeinsamen Betrachtung von verschiedenen Diagnosen gefunden. Auch die Häufigkeit des bipolaren Spektrums spricht hier dafür, dass sich die Natur nicht unbedingt an die diagnostischen Einschätzungen von DSM IV/V oder ICD10 hält.

Prof. Dr. Helge Frieling

Mesman E et al. The Dutch bipolar offspring study: 12-year follow-up. Am J Psychiatry 2013 170: $542-49$ 